RESEARCH ARTICLE

\title{
Exploration of Potential Bacterial Endophytes against Root Knot Nematode, Meloidogyne incognita in Banana
}

\author{
Ganeshan $K^{1}$, Vetrivelkalai $P^{1 *}$, Bhagawati $B^{2}$, Nibha $G^{3}$, Devrajan $K^{1}$, Raveendran $M^{4}$ and Balachander $D^{5}$ \\ ${ }^{1}$ Department of Nematology, Tamil Nadu Agricultural University, Coimbatore- 641003 \\ 2 Department of Nematology, Assam Agricultural University, Jorhat, Assam-785013 \\ ${ }^{3}$ Division of Plant Pathology and Microbiology, Regional Plant Resource Centre, Bhubaneswar, Odisha-751015 \\ ${ }^{4}$ Department of Plant Biotechnology, Tamil Nadu Agricultural University, Coimbatore-641003 \\ ${ }^{5}$ Department of Agricultural Microbiology, Tamil Nadu Agricultural University, Coimbatore-641003
}

\begin{abstract}
A field survey was conducted in 12 districts of Assam viz., Jorhat, Golaghat, Nagaon, Marigaon, Goalpara, Dibrugarh, Tinsukia, Lakhimpur, Dhemaji, Sivsagar, Kamrup and Barpeta. A total of 92 root samples were collected and 37 bacterial isolates were isolated from commercial banana cultivars. The culture filtrates extracted from 37 endophytic bacterial isolates, were screened against southern root-knot nematode, Meloidogyne incognita in vitro and under pot culture studies. The five bacterial isolates viz., EB4, EB8, $\mathrm{BC} 1, \mathrm{BC} 11$ and $\mathrm{BC} 12$ showed $100 \%$ inhibition of egg hatching and juvenile mortality of $M$. incognita with an exposure period of 48 and $72 \mathrm{~h}$. On seed bacterization, with these five promising isolates, two isolates viz.EB4, BC1 significantly enhanced germination percentage $(33.33,25.31 \%)$ and vigour index $(75.5,64.39 \%)$ of paddy, receptively. The potential bacterial isolates viz., BC1 and EB4 were identified as Lysinibacillus sp. and Pseudomonas sp., respectively , based on the morphological phenotypic and biochemical characterization. The pot culture experiment revealed that the bacterial endophytes viz., Lysinibacillus sp. (BC1) Pseudomonas sp. (EB4) significantly reduced the soil $(61.64,56.71 \%)$ and root nematode population $(77.29$, $68.87 \%)$, number of adult females $(73.97,69.89 \%)$, egg masses $(85.63$, $80.11 \%)$ and root-knot index $(1.33,1.67)$ of $M$. incognita compared to untreated control. The bacterial endophytes viz., Pseudomonas sp. (EB4), Lysinibacillus sp. (BC1) were also significantly increased the growth parameters viz., shoot length $(43.33,39.18 \%)$, and root length $(78.24$, $59.26 \%$ ) and pesudostem girth (58.38, 52.13\%).
\end{abstract}

Keywords: Lysinibacillus sp; Pseudomonas sp.; Endophytes; antinemic property; Banana; Root-knot nematode and Growth promotion.

\section{INTRODUCTION}

Root-knot nematode, Meloidogyne spp. are obligate, sedentary endoparasites of more than 3000 host plants throughout the world (Sasser, 1979). The southern root-knot nematode, $M$. incognita is one of the major constraints in the production of banana and caused the $15 \%$ yield loss in India (Kumar et al., 2020). The main symptom of infestations is typical terminal or tip galls formation and distorted root architecture that attacks the primary and secondary roots (Faske et al., 2018). In recent years, the use of chemical nematicide decreased due to the effective utilization of antagonistic bio-control agents. Plants have complex micro-ecosystems in which different niches are filled by a wide variety of beneficial microorganisms
(Souza et al., 2013). Bacterial endophytes act as biocontrol agents for nematodes (Jie et al., 2009) and promote plant growth in various crops (Kausal et al., 2017). Presently, most commercial biocontrol products on the market contain live microorganisms, such as Pasteuria penetrans, Purpureocillium lilacinum, and/or their metabolites, which target specific nematodes (Lamovsek et al., 2013).

In general, the phyllosphere region has /more microbial community; however, the root region also has bacterial endophytes, which share same niche with M. incognita (Abdel-Salam et al., 2018). Endophytes colonized the plant root tissues may manage sedentary endoparasitic nematodes because both occupy the same ecological niche and are in close association throughout the nematode life 
cycle, which makes these bacteria act as excellent biocontrol agent. The endophytes also elicit signals for the induction of defense mechanisms against root-knot nematode. Hence, the present study aims to test the antinemic properties like ovicidal and larvicidal of bacterial endophytes isolated from banana root against root-knot nematode, $M$. incognita and also test their potential for growth enhancement.

\section{MATERIALS AND METHODS}

A field survey was conducted to collect the healthy banana roots for the isolation of endophytes from 12 districts of Assam viz., Jorhat, Golaghat, Nagaon, Marigaon, Goalpara, Dibrugarh, Tinsukia, Lakhimpur, Dhemaji, Sivsagar, Kamrup and Barpeta. A total of 92 root samples were collected from different banana cultivars viz., Malbhog, Seni Champa and Jahaji. The young feeder roots were collected at a depth of 20 to $30 \mathrm{~cm}$ and collected samples were packed in properly labeled zip lock cover then brought to the laboratory for bacterial isolation. In vitro and pot culture studies were conducted at the Department of Nematology, TNAU, Coimbatore.

\section{Isolation of endophytic bacteria and identification}

The collected root samples were washed with tap water and cut into small pieces $(1-2 \mathrm{~cm})$ using a sterile blade. Root samples were surface sterilized with $5 \% \mathrm{NaOCl}$ for 20 minutes, then washed twice with sterile distilled water to remove excess $\mathrm{NaOCl}$. Subsequently washed with 70 per cent ethanol for 30 seconds and then washed 8 times with sterile distilled water, from the $8^{\text {th }}$ wash $1 \mathrm{~mL}$ was drawn and plated in Nutrient Agar medium as a sterile check. The root samples were homogenized using pestle and mortar by sterile peptone salt ( $1 \mathrm{~g}$ of peptone $+1 \mathrm{~g}$ of $\mathrm{NaCl}$ in 1 litre of sterile distilled water) 10 $\mathrm{mL}$ was used for maceration of a sample, allow it to withstand for 20 minutes and $1 \mathrm{~mL}$ of supernatant was taken from each sample for serial dilution. Three dilutions were carried out serially $\left(10^{6}\right.$ to $\left.10^{8}\right)$ for each sample and spread on the Nutrient Agar (NA). Two replications were maintained for each dilution. The NA plates were incubated at $37^{\circ} \mathrm{C}$ for week $3-5$ days for bacterial colony growth. The isolates were initially categorized into two broad groups based on the Gram staining technique described in Hucker's modified method (Rangaswami and Bagyaraj, 1993). Morphological and cultural characters of the isolates were used for further grouping. Based on the results of various biochemical tests viz., starch hydrolysis, $\mathrm{KOH}$ test, citrate utilization, catalase, methyl red, gelatin hydrolysis, growth at $4^{\circ} \mathrm{C}$, the organisms were identified upto generic level by Bergey's manual and Vetrivelkalai et al. (2010).

\section{In vitro screening of bacterial endophytes against $M$. incognita}

Endophytic bacterial isolates were grown on nutrient broth and allowed for incubation. The culture filtrate was obtained by centrifuging the broth at $10000 \mathrm{rpm}$ for 15 minutes and passed through $0.2 \mu \mathrm{m}$ bacterial filter to avoid the cells and spores. A total of 37 bacterial isolates were screened against $M$. incognita under laboratory conditions. The effect on egg hatching and juvenile mortality of $M$. incognita were done in vitro using 2 $\mathrm{mL}$ of cell-free culture filtrate. The eggs and J2 of $M$. incognita were placed in a cavity block (100 nos.) and incubated at $27 \pm 2{ }^{\circ} \mathrm{C}$ for studying egg hatching and juvenile mortality, respectively. The broth without bacteria and distilled water were used as control. Observations were recorded on number of egg hatched and immobilized juveniles after 24, 48 and $72 \mathrm{~h}$ of incubation (Vetrivelkalai and Sivakumar, 2019). Three replications were maintained for each isolate and the experiment was arranged in a completely randomized design.

\section{Testing of growth promotion}

Among the 37 bacterial isolates, five promising bacterial endophytes were used for testing the growth promotion activities. Seed bacterization was done by soaking paddy seeds ( 25 numbers) in small petri dish $(5 \mathrm{~mL})$, which contains $3 \mathrm{~mL}$ of bacterial culture and assessed by a modified roll towel method (ISTA, 1993). The germination percentage, shoot length and root length was recorded at 25 days after germination. The Vigour index (VI) was calculated using the following formula (Abdul Baki and Anderson, 1973). VI = Germination percentage $X$ Seedling length (shoot length + root length).

\section{Evaluation of bacterial endophytes against $M$. incognita in banana}

The pot mixtures containing red soil, sand and FYM @ 2:1:1 ratio were prepared and sterilized in an autoclave and was filled in earthen pot. The banana cv. Ney Poovan suckers were trimmed and dipped with bacterial culture @ 10mL/sucker for 15-20 minutes, then planted in $5 \mathrm{~kg}$ earthen pots at one sucker per pot. Freshly hatched $5000 \mathrm{~J} 2$ of $M$. incognita was inoculated per pot at 15 days after planting. Three replications were maintained for each isolate and the experiment was arranged in a completely randomized design. The observations on growth parameters and nematode multiplication factors were recorded at 90 days after nematode inoculation. The collected soil and root samples were processed by Cobb's decanting and sieving method (Cobb, 1918) and Modified Baermann funnel technique (Schindler, 1961), respectively. The representative $5 \mathrm{~g}$ root samples of each pot were washed free of soil and stained with 0.1 per cent 
acid fuchsin - lactophenol to examine the number of females and egg masses. The root-knot index was graded using 1 to 5 scale rating (Heald et al., 1989) viz., 1: no galls; 2: 1-25\% galls; 3: $25-50 \%$ galls 4: $50-75 \%$ galls; 5 ; > 75\% galls/ root system.

\section{Statistical analysis}

All the experiments were analyzed independently. The treatment means were compared by Duncan's Multiple Range-Test (DMRT) (Gomez and Gomez, 1984). The package used for analysis was IRRISTAT version 92-1 developed by the International Rice Research Institute Biometrics unit, Philippines.

\section{RESULTS AND DISCUSSION}

\section{Survey, isolation and identification of endophytic bacteria}

A total of 37 endophytic bacterial isolates were obtained from different banana cultivars viz., Malbhog, Seni Champa and Jahaji. Isolation of endophytic bacterial strains from various monocots and woody plants has reported by several authors (Ragavi et al., 2019; Kaushal et al., 2020 and Gomez-Lama Cabanas et al. (2021).

\section{In vitro screening against nematodes}

Among the bacterial isolates screened, 12 isolates viz., EB4, EB8 EA1, EA2, EA3, EA4, EA5, BC1, $\mathrm{BC} 4, \mathrm{BC} 9, \mathrm{BC} 11$ and $\mathrm{BC} 12$ found that inhibited egg hatching of $M$. incognita by cent per cent (100\%) with an exposure period of 24,48 and 72 h at 100 per cent concentration of culture filtrate. The second stage juvenile of M.incognita was dead at 100 per cent in 10 bacterial isolates viz., EB1, EB2, EB3, EB4, EB5, EB8, EB9, BC1, BC11 and BC12 at 48 and $72 \mathrm{~h}$ exposure period (Table1).

Among these 37 bacterial isolates, five isolates viz., EB4 BC8, BC1, BC11 and $\mathrm{BC} 12$ possessed both inhibitions of egg hatching and juvenile mortality of M. incognita with an exposure period of 48 and $72 \mathrm{~h}$ at 100 per cent concentration of cell free culture filtrate. The high degree of ovicidal and larvicidal properties of the endophytic bacterial isolates is attributed due to parasitizing, production of the toxin, secondary metabolites and antibiotics. The findings of Munif et al., (2019) were in accordance with the present study in which they have reported endophytic consortium inhibited $M$. incognita egg hatching up to $81.33 \%$ and increased J2 of M. incognita mortality up to 85 per cent compared to control. Su et al. (2017) Streptomyces sp. strain showed an inhibiting rate of $>50$ per cent in vitro and biocontrol efficiency of 70.7 per cent against M. javanica compared to the control.

Table 1. Effect of culture filtrate of various isolates of bacterial endophytes on egg hatching and juvenile mortality of $M$. incognita

\begin{tabular}{|c|c|c|c|c|c|c|}
\hline \multirow{2}{*}{$\begin{array}{c}\text { Endophytic } \\
\text { bacterial } \\
\text { isolates }\end{array}$} & \multicolumn{3}{|c|}{ Number of eggs hatched } & \multicolumn{3}{|c|}{ Number of dead juveniles } \\
\hline & $24 \mathrm{~h}$ & $48 h$ & $72 \mathrm{~h}$ & $24 \mathrm{~h}$ & $48 h$ & $72 \mathrm{~h}$ \\
\hline EB1 & $1.67^{\mathrm{cd}}(1.27)$ & $3.67^{\text {cd }}(2.04)$ & $8.33^{\circ}(2.97)$ & $5.00^{\mathrm{kl}}(2.35)$ & $100.00^{\mathrm{a}}(10.02)$ & $100.00^{a}(10.02)$ \\
\hline EB2 & $1.33^{\mathrm{bc}}(1.35)$ & $2.67^{\mathrm{bc}}(1.78)$ & $9.67^{\circ}(3.19)$ & $1.67^{\mathrm{op}}(1.47)$ & $100.00^{a}(10.02)$ & $100.00^{a}(10.02)$ \\
\hline EB3 & $1.00^{\mathrm{b}}(1.22)$ & $1.67^{\mathrm{b}}(1.47)$ & $5.33^{\mathrm{b}}(2.42)$ & $4.67^{\mathrm{klm}}(2.27)$ & $100.00^{\mathrm{a}}(10.02)$ & $100.00^{\mathrm{a}}(10.02)$ \\
\hline EB4 & $0.00^{\mathrm{a}}(0.71)$ & $0.00^{\mathrm{a}}(0.71)$ & $0.00^{\mathrm{a}}(0.71)$ & $100.00^{\mathrm{a}}(10.02)$ & $100.00^{a}(10.02)$ & $100.00^{\mathrm{a}}(10.02)$ \\
\hline EB5 & $1.00^{\mathrm{b}}(1.22)$ & $6.00^{\mathrm{e}}(2.55)$ & $12.00^{d}(3.54)$ & $18.67^{e}(4.38)$ & $100.00^{\mathrm{a}}(10.02)$ & $100.00^{a}(10.02)$ \\
\hline EB6 & $3.33^{\text {ef }}(1.96)$ & $9.33^{f}(3.14)$ & $22.00^{\text {gh }}(4.74)$ & $4.33^{\mathrm{klmn}}(2.20)$ & $12.33^{k}(3.58)$ & $21.33^{\mathrm{mn}}(4.67)$ \\
\hline EB7 & $3.67^{f}(2.04)$ & $5.00^{\text {de }}(2.35)$ & $8.00^{\circ}(2.92)$ & $3.00^{\text {no }}(1.87)$ & $7.67^{1}(2.86)$ & $20.00^{\mathrm{mno}}(4.53)$ \\
\hline $\mathrm{BC} 8$ & $0.00^{\mathrm{a}}(0.71)$ & $0.00^{\mathrm{a}}(0.71)$ & $0.00^{\mathrm{a}}(0.71)$ & $100.00^{\mathrm{a}}(10.02)$ & $100.00^{\mathrm{a}}(10.02)$ & $100.00^{a}(10.02)$ \\
\hline EB9 & $4.33^{d}(2.20)$ & $12.67^{\text {hi }}(3.63)$ & $13.33^{\mathrm{de}}(3.72)$ & $1.33^{\mathrm{pq}}(1.35)$ & $100.00^{\mathrm{a}}(10.02)$ & $100.00^{a}(10.02)$ \\
\hline EB10 & $7.00^{i}(2.74)$ & $14.67^{\mathrm{k}}(3.89)$ & $20.33^{g}(4.56)$ & $8.00^{i j}(2.92)$ & $17.33^{j}(4.22)$ & $21.67^{\mathrm{m}}(4.71)$ \\
\hline EB11 & $8.33^{j}(2.97)$ & $17.67^{\prime}(4.26)$ & $28.67^{j}(5.40)$ & $10.67^{\mathrm{h}}(3.34)$ & $17.33^{\mathrm{j}}(4.22)$ & $23.00^{\operatorname{lm}}(4.85)$ \\
\hline EB12 & $4.33^{d}(2.20)$ & $11.33^{\text {gh }}(3.44)$ & $25.00^{i}(5.05)$ & $3.33^{m}(1.96)$ & $9.67^{\prime}(3.19)$ & $17.33^{\circ p}(4.22)$ \\
\hline EA1 & $0.00^{a}(0.71)$ & $0.00^{a}(0.71)$ & $0.00^{a}(0.71)$ & $0.00^{a}(0.71)$ & $3.33^{m n}(1.96)$ & $6.33 r(2.61)$ \\
\hline EA2 & $0.00^{\mathrm{a}}(0.71)$ & $0.00^{a}(0.71)$ & $0.00^{\mathrm{a}}(0.71)$ & $0.00^{a}(0.71)$ & $9.33^{\prime}(3.14)$ & $17.67^{\text {nop }}(4.26)$ \\
\hline EA3 & $0.00^{\mathrm{a}}(0.71)$ & $0.00^{\mathrm{a}}(0.71)$ & $0.00^{\mathrm{a}}(0.71)$ & $0.00^{\mathrm{q}}(0.71)$ & $0.33^{\circ}(0.91)$ & $1.00^{\mathrm{s}}(1.22)$ \\
\hline EA4 & $0.00^{\mathrm{a}}(0.71)$ & $0.00^{a}(0.71)$ & $0.00^{\mathrm{a}}(0.71)$ & $0.00^{a}(0.71)$ & $0.00^{\circ}(0.71)$ & $1.00^{\mathrm{s}}(1.22)$ \\
\hline EA5 & $0.00^{\mathrm{a}}(0.71)$ & $0.00^{\mathrm{a}}(0.71)$ & $0.00^{\mathrm{a}}(0.71)$ & $0.00^{\mathrm{q}}(0.71)$ & $0.67^{\circ}(1.08)$ & $\left.1.33^{s} 1.35\right)$ \\
\hline $\mathrm{BC} 1$ & $0.00^{a}(0.71)$ & $0.00^{a}(0.71)$ & $0.00^{a}(0.71)$ & $100.00^{\mathrm{a}}(10.02)$ & $100.00^{\mathrm{a}}(10.02)$ & $100.00^{\mathrm{a}}(10.02)$ \\
\hline $\mathrm{BC} 2$ & $3.67^{f}(2.04)$ & $13.00^{\mathrm{ij}}(3.67)$ & $20.00^{8}(4.53)$ & $9.33^{\text {hi }}(3.14)$ & $20.67^{i}(4.60)$ & $28.00^{k}(5.34)$ \\
\hline $\mathrm{BC} 3$ & $2.67^{\text {de }}(1.78)$ & $11.33^{\text {gh }}(3.44)$ & $15.00^{\mathrm{ef}}(3.94)$ & $16.00^{f}(4.06)$ & $27.67^{f}(5.31)$ & $33.00^{\text {hi }}(5.79)$ \\
\hline
\end{tabular}




\begin{tabular}{|c|c|c|c|c|c|c|}
\hline BC4 & $0.00^{\mathrm{a}}(0.71)$ & $0.00^{\mathrm{a}}(0.71)$ & $0.00^{\mathrm{a}}(0.71)$ & $13.33^{g}(3.72)$ & $22.67^{\text {hi }}(4.81)$ & $26.00^{k l}(5.15)$ \\
\hline BC5 & $14.33^{p}(3.85)$ & $22.00^{n}(4.74)$ & $25.00^{i}(5.05)$ & $3.00^{n}(1.87)$ & $9.67^{\prime}(3.19)$ & $5.67^{p}(4.02)$ \\
\hline BC6 & $3.67^{\mathrm{fg}}(2.04)$ & $10.33^{\mathrm{fg}}(3.29)$ & $14.33^{\mathrm{e}}(3.85)$ & $13.00^{g}(3.67)$ & $16.00^{f g}(5.15)$ & $32.33^{\mathrm{j}}(5.73)$ \\
\hline $\mathrm{BC} 7$ & $11.67^{\mathrm{kl}}(3.49)$ & $20.33^{m}(4.56)$ & $23.67^{\text {hi }}(4.92)$ & $3.67^{\operatorname{mn}}(2.04)$ & $13.33^{k}(3.72)$ & $20.67^{a}(4.60)$ \\
\hline $\mathrm{BC} 8$ & $16.33^{9}(4.10)$ & $26.67^{p}(5.21)$ & $30.00^{j}(5.52)$ & $1.33^{9}(1.35)$ & $5.00^{m}(2.35)$ & $11.00(3.39)$ \\
\hline BC9 & $0.00^{a}(0.71)$ & $0.00^{\mathrm{a}}(0.71)$ & $0.00^{\mathrm{a}}(0.71)$ & $13.33^{g}(3.72)$ & $23.67^{\mathrm{gh}}(4.92)$ & $29.33^{\mathrm{jk}}(5.46)$ \\
\hline BC10 & $3.00^{\text {ef }}(1.87)$ & $10.00^{\text {fg }}(3.24)$ & $13.33^{\text {de }}(3.72)$ & $5.33^{\mathrm{k}}(2.42)$ & $14.67^{\mathrm{jk}}(3.89)$ & $22.67^{\mathrm{Im}}(4.81)$ \\
\hline BC11 & $0.00^{\mathrm{a}}(0.71)$ & $0.00^{a}(0.71)$ & $0.00^{\mathrm{a}}(0.71)$ & $100.00^{\mathrm{a}}(10.02)$ & $100.00^{\mathrm{a}}(10.02)$ & $100.00^{\mathrm{a}}(10.02)$ \\
\hline $\mathrm{BC} 12$ & $0.00^{\mathrm{a}}(0.71)$ & $0.00^{\mathrm{a}}(0.71)$ & $0.00^{\mathrm{a}}(0.71)$ & $100.00^{\mathrm{a}}(10.02)$ & $100.00^{\mathrm{a}}(10.02)$ & $100.00^{\mathrm{a}}(10.02)$ \\
\hline $\mathrm{BC} 13$ & $6.00^{h}(2.55)$ & $13.00^{\mathrm{ij}}(3.67)$ & $17.33^{f}(4.22)$ & $38.67^{\mathrm{b}}(6.26)$ & $79.67^{b}(8.95)$ & $90.33^{b}(9.53)$ \\
\hline BC14 & $12.00^{\operatorname{Im}}(3.54)$ & $23.33^{\text {no }}(4.88)$ & $36.33^{\prime}(6.07)$ & $21.33^{d}(4.67)$ & $38.67^{d}(6.26)$ & $73.67^{d}(8.61)$ \\
\hline BC15 & $11.00^{k}(3.39)$ & $19.00^{\mathrm{Im}}(4.42)$ & $36.67^{\prime}(6.10)$ & $18.67^{e}(4.38)$ & $35.67^{\mathrm{e}}(6.01)$ & $66.67^{\mathrm{e}}(8.20)$ \\
\hline BC16 & $12.67^{\mathrm{mn}}(3.63)$ & $24.00^{\circ}(4.49)$ & $41.67^{m}(6.49)$ & $4.67^{\mathrm{klm}}(2.27)$ & $16.67^{\mathrm{j}}(4.14)$ & $36.00^{\text {gh }}(6.04)$ \\
\hline BC17 & $12.33^{\operatorname{lmn}}(3.58)$ & $18.00^{\prime}(4.30)$ & $34.00^{k}(5.87)$ & $7.67^{\mathrm{j}}(2.86)$ & $20.67^{i}(4.60)$ & $36.33^{\text {gh }}(6.04)$ \\
\hline BC18 & $13.67^{\mathrm{op}}(3.76)$ & $24.33^{\circ}(4.98)$ & $30.00^{j}(5.52)$ & $5.00^{\mathrm{kl}}(2.35)$ & $20.67^{i}(4.60)$ & $39.00^{g}(6.28)$ \\
\hline BC19 & $8.67^{j}(3.03)$ & $14.33^{j k}(3.85)$ & $21.00^{g}(4.64)$ & $27.33^{\mathrm{c}}(5.28)$ & $52.33^{\circ}(7.27)$ & $83.00^{\circ}(9.14)$ \\
\hline BC2O & $13.00^{\text {no }}(3.67)$ & $19.33^{\operatorname{lm}}(4.45)$ & $29.33^{j}(5.46)$ & $13.33^{8}(3.72)$ & $27.33^{f}(5.28)$ & $50.33^{f}(7.13)$ \\
\hline Nutrient broth & $45.00^{r}(6.75)$ & $76.33^{9}(8.73)$ & $90.00^{n}(9.48)$ & $0.00^{q}(0.71)$ & $0.00^{\circ}(0.71)$ & $0.00^{\mathrm{s}}(0.71)$ \\
\hline Distilled water & $49.00^{\mathrm{s}}(7.04)$ & $80.67^{r}(8.93)$ & $92.83^{\circ}(9.63)$ & $0.00^{\circ}(0.71)$ & $0.00^{\circ}(0.71)$ & $0.00^{\mathrm{s}}(0.71)$ \\
\hline SEd & 0.50 & 0.73 & 0.85 & 0.72 & 1.26 & 1.68 \\
\hline$C D(P=0.01)$ & 1.33 & 1.93 & 2.25 & 1.92 & 3.34 & 4.44 \\
\hline
\end{tabular}

*Values are mean of three replications

Means followed by a common letter are not significantly different at $1 \%$ level by DMRT

Values in parenthesis are square root transformed values

\section{Morphology and biochemical characterization}

Based on the results of egg hatching and juvenile mortality, five isolates were identified using biochemical methods. The Gram stain reaction and cell morphology studies revealed that out of five isolates, three isolates EB4 to EB8 and BC11 belonged to Gram-negative and rod-shaped, nonspore-forming bacteria and two isolates viz., BC1 and BC12 belonged to Gram-positive and rod-shaped, spore-forming bacteria. The results obtained by analyzing primary character and nutrient source utilization of different endophytic bacteria revealed that three endophytic bacterial isolates viz., EB4 and BC11 belonged to Pseudomonas spp., EB8 belonged to Klebsiella sp. and two bacterial isolates viz., BC1 belonged to Lysinibacillus and BC12 belonged to Bacillus spp. (Table 2). This result is in confirmation with the findings of Vetrivelkalai et al. (2010), who have recorded that based on the positive and negative results of different biochemical test, production of metabolites and utilization of nutrient sources, ten endophytic bacterial isolates viz., EB9 to EB18 identified as Bacillus spp. and nine isolates EB1 to EB8 and EB19 identified as Pseudomonas spp.

Table 2. Biochemical characterization of endophytic bacterial isolates

\begin{tabular}{|c|c|c|c|c|c|c|c|c|c|c|}
\hline S. No & $\begin{array}{c}\text { Endophytic } \\
\text { isolates }\end{array}$ & $\begin{array}{c}\text { Gram } \\
\text { staining }\end{array}$ & $\begin{array}{c}\text { Catalase } \\
\text { test }\end{array}$ & $\begin{array}{l}\text { KOH } \\
\text { test }\end{array}$ & $\begin{array}{c}\text { Citrate } \\
\text { utilization }\end{array}$ & $\begin{array}{c}\text { Growth in } \\
\mathbf{5 \%} \mathrm{NaCl}\end{array}$ & $\begin{array}{c}\text { Starch } \\
\text { hydrolysis }\end{array}$ & $\begin{array}{c}\text { IAA } \\
\text { production }\end{array}$ & $\begin{array}{c}\text { Flourescence on } \\
\text { KB medium }\end{array}$ & $\begin{array}{l}\text { Genus } \\
\text { name }\end{array}$ \\
\hline 1 & EB4 & - & + & + & + & + & - & + & + & Pseudomonas sp. \\
\hline 2 & EB8 & - & + & + & + & - & + & + & + & Klebsiella sp. \\
\hline 3 & $\mathrm{BC} 1$ & + & + & - & + & + & + & + & - & Lysinibacillus sp. \\
\hline 4 & $\mathrm{BC} 11$ & - & + & + & + & - & - & - & + & Pseudomonas sp. \\
\hline 5 & $\mathrm{BC} 12$ & + & + & - & + & + & - & - & - & Bacillus sp. \\
\hline
\end{tabular}

\section{Growth promotion activity}

The results of the current study revealed that seed bacterization with endophytic bacterial isolates viz., EB4 and BC1 enhanced vigour index in paddy by 75.5 and 64.39 per cent, respectively (Fig. 1).
Germination percentage (33.33; $25.31 \%)$, root length $(49.82 ; 36.55 \%)$ and shoot length $(63.85$; $53 \%)$ were increased compared to untreated control. Similarly, various reports have indicated that bacterial endophytes promoted the growth and 
health of crop plants (Vetrivelkalai and Sivakumar, 2019; Munif et al. 2019). The mechanisms by which growth improved may be the production of phytohormones, IAA and enhanced availability of nutrients, reduction of ethylene levels, production of antibiotics and induced systemic resistance. Antagonism bacterial endophyte improves the resistance and exhibited PGPR activities (Liu et al., 2019; Bubici et al., 2019). The present results were also in conformity with the earlier reports.

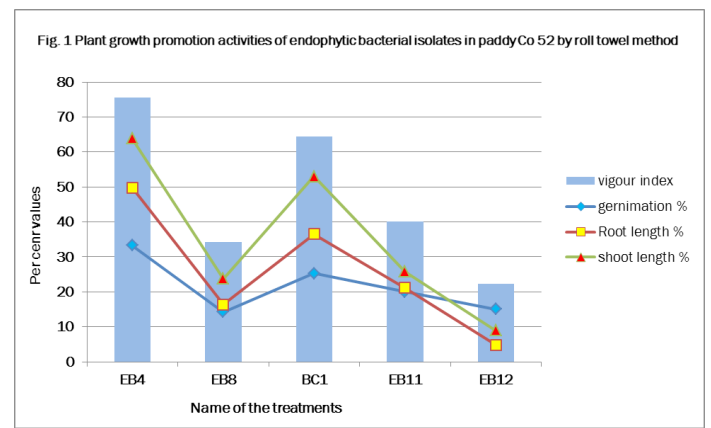

Table 3. Influence of endophytes on growth parameters of banana under pot culture conditions

\begin{tabular}{|c|c|c|c|c|c|}
\hline Endophytic bacteria & Shoot length (cm) & Root length (cm) & Shoot weight (g) & Root weight (g) & Pseudostem grid (cm) \\
\hline \multirow{2}{*}{ Pseudomonas sp. (EB4) } & $57.33^{a}$ & $128.33^{a}$ & $322.67^{a}$ & $486.67^{a}$ & $12.67^{a}$ \\
\hline & (43.33) & $(78.24)$ & $(40.29)$ & $(82.27)$ & $(58.38)$ \\
\hline \multirow{2}{*}{ Klebsiella sp. (EB8) } & $52.67^{b}$ & $111.33^{b}$ & $265.67^{c}$ & $340.67^{b}$ & $11.00^{\mathrm{b}}$ \\
\hline & $(24.06)$ & (35.33) & $(13.43)$ & $(21.63)$ & $(27.27)$ \\
\hline \multirow{2}{*}{ Lysinibacillus sp. (BC1) } & $55.67^{a}$ & $114.67^{b}$ & $298.67^{b}$ & $457.67^{b}$ & $12.17^{\mathrm{a}}$ \\
\hline & (39.18) & $(59.26)$ & $(29.86)$ & $(71.41)$ & (52.13) \\
\hline \multirow{2}{*}{$\begin{array}{l}\text { Pseudomonas sp. } \\
\text { (BC11) }\end{array}$} & $53.67^{b}$ & $108.67^{b}$ & $257.33^{c}$ & $328.33^{b}$ & $10.83^{b}$ \\
\hline & (34.18) & $(50.93)$ & $(29.86)$ & $(22.97)$ & $(35.38)$ \\
\hline \multirow{2}{*}{ Bacillus sp. (BC12) } & $49.67^{b}$ & $96.33^{c}$ & $251.33^{c}$ & $314.00^{b}$ & $10.17^{c}$ \\
\hline & $(19.47)$ & $(25.26)$ & $(11.88)$ & $(14.97)$ & $(21.34)$ \\
\hline Nutrient broth & $39.00^{c}$ & $72.33^{d}$ & $236.33^{d}$ & $267.67^{c}$ & $8.83^{c}$ \\
\hline Distilled water Control & $40.33^{c}$ & $74.33^{d}$ & $239.00^{d}$ & $268.67^{c}$ & $8.53^{c}$ \\
\hline SEd & 1.06 & 2.80 & 5.88 & 7.57 & 0.25 \\
\hline$C D(P=0.01)$ & 3.18 & 8.33 & 17.52 & 22.56 & 0.75 \\
\hline
\end{tabular}

${ }^{*}$ Values are mean of three replications

Means followed by a common alphabet of not significantly different at $1 \%$ level by DMRT

Values in parenthesis are per cent increased over control

\section{Evaluation of bacterial endophytes against M. incognita in banana}

The best performing five bacterial isolates screened for their nematicidal action against rootknot nematode, $M$. incognita in banana based on the results of growth promotion activities. The significant reduction in the number of adult females and number of egg masses was observed in banana plants treated with Lysinibacillus (BC1) isolate by 73.97 and 85.63.It was followed by Pseudomonas (EB4) $(69.86 ; 80.11 \%)$.

Table 4. Effect of endophytes on root-knot nematode multiplication under pot culture conditions

\begin{tabular}{|c|c|c|c|c|c|}
\hline Endophytic bacteria & $\begin{array}{l}\text { Soil population } \\
\text { (200 cc) }\end{array}$ & $\begin{array}{c}\text { Root population } \\
(5 g)\end{array}$ & $\begin{array}{c}\text { No. of females } \\
(5 \mathrm{~g})\end{array}$ & $\begin{array}{l}\text { No. of egg masses } \\
(5 g)\end{array}$ & $\begin{array}{l}\text { Root-knot } \\
\text { index }\end{array}$ \\
\hline \multirow{2}{*}{ Pseudomonas sp. (EB4) } & $172.00^{b}$ & $28.33^{b}$ & $22.00^{a}$ & $12.00^{b}$ & \multirow{2}{*}{1.67} \\
\hline & $(56.71)$ & $(68.87)$ & $(69.86)$ & $(80.11)$ & \\
\hline \multirow{2}{*}{ Klebsiella sp. (EB8) } & $203.67^{c}$ & $63.33^{d}$ & $57.67^{e}$ & $29.00^{\mathrm{e}}$ & \multirow{2}{*}{4.0} \\
\hline & $(48.74)$ & $(30.41)$ & $(21.00)$ & $(51.93)$ & \\
\hline \multirow{2}{*}{ Lysinibacillus sp. (BC1) } & $154.00^{\mathrm{a}}$ & $20.67^{a}$ & $19.00^{a}$ & $8.67^{a}$ & \multirow{2}{*}{1.33} \\
\hline & $(61.24)$ & $(77.29)$ & $(73.97)$ & $(85.63)$ & \\
\hline \multirow{2}{*}{ Pseudomonas sp. (BC11) } & $222.00^{d}$ & $49.67^{c}$ & $45.67^{\circ}$ & $21.00^{c}$ & \multirow{2}{*}{2.67} \\
\hline & $(44.13)$ & $(45.42)$ & (37.44) & (65.19) & \\
\hline \multirow{2}{*}{ Bacillus sp. (BC12) } & $216.33^{d}$ & $61.00^{d}$ & $52.00^{d}$ & $23.33^{d}$ & \multirow{2}{*}{3.0} \\
\hline & $(45.55)$ & $(32.97)$ & $(28.77)$ & (61.33) & \\
\hline Nutrient broth & $387.67^{\mathrm{e}}$ & $86.67^{\mathrm{e}}$ & $63.67^{f}$ & $53.33^{f}$ & 5.0 \\
\hline Distilled water Control & $397.33^{e}$ & $91.00^{f}$ & $73.00^{g}$ & $60.33^{g}$ & 5.0 \\
\hline SEd & 5.36 & 0.97 & 1.18 & 0.47 & - \\
\hline$C D(P=0.01)$ & 15.97 & 2.90 & 3.51 & 1.40 & - \\
\hline
\end{tabular}

*Values are mean of three replications

Means followed by a common alphabet of not significantly different at $1 \%$ level by DMRT

Values in parenthesis are per cent decreased over control 
The reduction in soil and root population observed in Lysinibacillus (BC1) treated plants by 61.24 and 77.29 per cent respectively, followed by Pseudomonas (EB4), which accounted for 56.71, 68.55 per cent reduction over control, respectively (Table 3 ). The lowest root gall index $(1.33 ; 1.67)$ registered in both Lysinibacillus (BC1) and Pseudomonas (EB4) treated banana plants compared to untreated control (5).

It was also found that banana suckers treated with Lysinibacillus (BC1) and Pseudomonas (EB4) significantly reduced the number of adult females, egg masses, and root and soil population of M. incognita under pot culture conditions. Vetrivelkalai (2019) and Munif et al. (2019) reported that endophytic bacterial isolates significantly reduced soil and root population, root gall index compared to untreated control. Siddiqui and Shaukat (2003) also found that cell suspension of $P$. fluorescens strains $\mathrm{CHAO}$ or CHAO/pME3424 at various inoculum levels $10^{7}, 10^{8}, 10^{9} \mathrm{cfu} / \mathrm{g}$ significantly reduced root knot development density in tomato under glasshouse conditions.

The mechanisms of nematode population reduction might be due to competition for space, nutrients; premature egg hatching, reduction in viability and mortality of juveniles induced by hydrocyanic acid, cellulase, lipases protease and $\beta$-1, 3-glucanase enzymes. Endophytic bacteria also triggered the production of indole acetic acid, enhanced the total nitrogen and available phosphorus (Naureen et al., 2017; Gopalakrishnan et al., 2015). The isolates viz., Pseudomonas (EB4) and Lysinibacillus (BC1) significantly increased growth parameters viz., pseudostem height, root length and pseudostem girth of banana under pot culture conditions (Table 4). The present results were also in conformity with the earlier reports of Harish (2005) found that application of endophytic bacterial strains significantly increased the growth parameters viz., pseudostem height, girth and number of leaves in banana plants under greenhouse conditions.

\section{CONCLUSION}

A varied group of microorganisms that play a significant role in optimizing plant growth and development inhabits plant roots. Endophytic bacteria viz., Lysinibacillus (BC1) and Pseudomonas (EB4) of banana have antinemic properties against root-knot nematode, $M$. incognita and growth promotion activities identified in the present study. Further, investigations on production of secondary metabolites and toxin compound, which is responsible for antinemic properties, have to be carried out. This will be helpful for the complete understanding and usage of endophytes as potential biocontrol agents for the management of nematodes.

\section{Funding and Acknowledgment}

We gratefully acknowledge the Department of Biotechnology, Govt. of India, New Delhi for providing the externally funded scheme as a network project under NER-BPMC-DBT-NER/AGRI/33/2016.

\section{Consent for publication}

All the authors agreed to publish the content.

\section{Competing interests}

There were no conflict of interest in the publication of this content

\section{REFERENCES}

Abdel-Salam, M. S., Ameen, H. H., Soliman, G. M., Elkelany, U. S., and A. M. Asar. 2018. Improving the nematicidal potential of Bacillus amyloliquefaciens and Lysinibacillus sphaericus against the root-knot nematode Meloidogyne incognita using protoplast fusion technique. Egypt. J. Biol. Pest Control., 28(1): 31.

Abdul Baki, A. A. and J. D. Anderson. 1973. Vigour determination in soybean seed by multiple criteria. Crop Sci., 31: 630-633.

Bubici, G., Kaushal, M., Prigigallo, M. I., Gomez-Lama Cabanas, C., and J. Mercado-Blanco. 2019. Biological control agents against Fusarium wilt of banana. Front. Microbiol., 10: 616.

Cobb, N. A. 1918. Estimating the nema population of soil, with special references to the sugarbeet and root-gall nemas, Heterodera schachtii Schmidt and Heterodera radicicola (Greef) Muller, and with a description of Tylencholaimus aequalis n. sp. Agric Tech Circular., 1: 48.

Faske, T. R., Overstreet, C., Lawrence, G., and T. L. Kirkpatrick. 2018. Important Plant Parasitic Nematodes of Row Crops in Arkansas, Lousiana and Mississippi. In Plant parasitic nematodes in sustainable agriculture of North America., 393431.

Gomez, K. A., and A. A. Gomez. 1984. Statistical procedures for agricultural research. John Wiley \& Sons.

Gomez-Lama Cabanas, C., Fernandez-Gonzalez, A. J., Cardoni, M., Valverde-Corredor, A., Lopez-Cepero, J., Fernandez-Lopez, M., and J. Mercado-Blanco. 2021. The banana root endophytome: Differences between mother plants and suckers and evaluation of selected bacteria to control Fusarium oxysporum f. sp. cubense. J. Fungus., 7(3): 194.

Gopalakrishnan, S., Srinivas, V., Prakash, B., Sathya, A., and R. Vijayabharathi. 2015. Plant growthpromoting traits of Pseudomonas geniculata isolated from chickpea nodules. 3 Biotech., 5(5): 653-661.

Harish, S. 2005. Molecular biology and diagnosis of banana buncy top virus and its management 
through induced systemic resistance. Ph. D., Thesis, TNAU, Coimbatore, India, 12p.

Heald, C. M., Bruton, B. D. and R.M. Davis. 1989. Influence of Glomus intradices and soil phosphorus on Meloidogyne incognita infecting Cucumis melo. J. Nematol., 21: 69-73.

ISTA, 1993. Proceedings of the International seed testing Association, International Rules for seed testing. Seed Sci. Technol., 21: 25-30.

Jie, L., Zifeng, W., Lixiang, C., Hongming, T., Patrik, I., Zide, J., and Z. Shining. 2009. Artificial inoculation of banana tissue culture plantlets with indigenous endophytes originally derived from native banana plants. Biol. Control., 51(3): 427-434.

Kaushal, M., Kumar, A., and R. Kaushal. 2017. Bacillus pumilus strain YSPMK11 as plant growth promoter and biocontrol agent against Sclerotinia sclerotiorum. 3 Biotech, 7(2): 90.

Kaushal, M., Swennen, R., and G. Mahuku. 2020. Unlocking the microbiome communities of banana (Musa spp.) under disease stressed (Fusarium wilt) and non-stressed conditions. Microorganisms, 8(3): 443.

Kumar, V., Khan, M. R., and R. K. Walia. 2020. Crop loss estimations due to plant parasitic nematodes in major crops in India. Natl. Acad. Sci. Lett., 43(5): 409-412.

Lamovsek, J., Urek, G., and S. Trdan. 2013. Biological control of root-knot nematodes (Meloidogyne spp.): microbes against the pests. Acta Agric. Slov., 101(2): 263-275.

Liu, Y., Zhu, A., Tan, H., Cao, L., and R. Zhang. 2019. Engineering banana endosphere microbiome to improve Fusarium wilt resistance in banana. Microbiome, 7(1): 74.

Munif, A., Supramana, S., Herliyana, E. N., and A. P. Pradana. 2019. Endophytic bacterial consortium originated from forestry plant roots and their nematicidal activity against Meloidogyne incognita infestation in greenhouse. Acta Universitatis Agriculturae Et Silviculturae Mendelianae Brunensis, 67(5):1171-1182.

Naureen, Z., Rehman, N. U., Hussain, H., Hussain, J., Gilani, S. A., Al Housni, S. K., and A. A. Harrasi. 2017. Exploring the potentials of Lysinibacillus sphaericus ZA9 for plant growth promotion and biocontrol activities against phytopathogenic fungi. Front. Microbiol., 8: 1477.

Ragavi, G., Muthamilan, M., Nakkeeran, S., Kumaravadivel, N., Sivakumar, U., and A. Suganthi. 2019. Phenotypic and Molecular Characterization of Endophytic Bacteria Isolated from Banana. Curr. J. Appl., 38(6): 1-10.

Rangaswami, G and D.J. Bagayaraj. 1993. Microbial Biotechnology. In: Agricultural Microbiology. Prentice Hall of India Pvt. Ltd., New Delhi. 389405.

Sasser, J. N. 1979. Economic importance of Meloidogyne in tropical countries. In: Lamberti F, Taylor CE (Eds.) Root knot nematodes (Meloidogyne species). Systematics, biology and control. Academic Press, New York, 359-373.

Schindler, A. F. 1961. A simple substitute for a Baermann funnel. Plant Disease Reporter., 45: 747-748.

Siddiqui, I. A., and S. S. Shaukat. 2003. Suppression of root knot disease by Pseudomonas fluorescens $\mathrm{CHAO}$ in tomato; importance of bacterial secondary metabolite, 2, 4-Diacetylpholoroglucinol. Soil Biol. Biochem., 35(12): 1615-1623.

Souza, S. A., Xavier, A. A., Costa, M. R., Cardoso, A., Pereira, M. C., and S. Nietsche. 2013. Endophytic bacterial diversity in banana - Prata Ana (Musa spp.) roots. Genet. Mol. Biol., 36(2): 252-264.

Su, L., Shen, Z., Ruan, Y., Tao, C., Chao, Y., Li, R., and Q. Shen. 2017. Isolation of antagonistic endophytes from banana roots against Meloidogyne javanica and their effects on soil nematode community. Front. Microbiol., 8: 2070.

Vetrivelkalai, P. 2019. Evaluation of endophytic bacterial isolates against root knot nematode, Meloidogyne incognita in tomato under glasshouse conditions. Int. J. Curr. Microbiol. Appl. Sci., 8: 2584-2589.

Vetrivelkalai, P. and M. Sivakumar. 2019. Characterization of antinematicidal and antifungal bacterial microbes by 16 s rRNA Gene sequence. Int. J. Curr. Microbiol. App. Sci, 8(1): 2575-2583.

Vetrivelkalai, P., Sivakumar, M. and Jonathan, E.I. 2010. Biocontrol potential of endophytic bacteria on Meloidogyne incognita and its effect on plant growth in bhendi. J. Biopestic. 3(2): 452-457. 